\title{
On Certain Analytic Functions
}

\section{Janusz Sokol ${ }^{2 *}$ and Mamoru Nunokawa ${ }^{1}$}

${ }^{1}$ University of Gunma, Hoshikuki-cho 798-8, Chuou-Ward, Chiba, 260-0808, Japan

${ }^{2}$ Department of Mathematics, Rzesz'ow University of Technology, Al. Powsta'nc'ow Warszawy 12, 35-959 Rzesz'ow, Poland

\author{
Abstract \\ We apply Nunokawa's lemma, On Properties of Non-Carath'eodory Functions, Proc. Japan Acad. 68, Ser. A \\ (1992) 152-153, to prove some new results.
}

Keywords: Analytic; Univalent; Convex; Starlike; Strongly starlike; Differential subordination

\section{Introduction}

For integer $\mathrm{n} \geq 0$, denote by $\Sigma \mathrm{n}$ the class of meromorphic functions, defined in $\dot{U}=\{\mathrm{z}: 0<|z|<1\}$, which are of the form

$$
F(\mathrm{z})=\frac{1}{z}+a_{n} z^{n}+a_{n+1} z^{n+1}+\ldots
$$

A function $\mathrm{F} € \Sigma_{0}$ is said to be starlike if it is univalent and the complement of $\mathrm{F}(\grave{\mathrm{U}})$ is starlike with respect to the origin. Denote by $\Sigma^{*}$ the class of such functions. If $\mathrm{F} € \Sigma_{0}$, then it is well-known that $\mathrm{F} €$ $\Sigma^{*}{ }_{0}$ if and only if

$$
\mathfrak{R} e\left\{-\frac{z F^{\prime}(\mathrm{z})}{F(\mathrm{z})}\right\}>0
$$

for $\mathrm{z} €$ Ù. For $\alpha<1$, let

$$
\sum_{n, \alpha}^{*}=\left\{F \in \sum_{n}: \mathfrak{R e}\left\{\frac{z F^{\prime}(\mathrm{z})}{F(\mathrm{z})}\right\}>\alpha, z \in \dot{U}\right\},
$$

The class of meromorphic-starlike functions of order $\alpha$. For $0<\alpha \leq$ 1 , let

$$
\sum_{n}^{*}(\alpha)=\left\{F \in \sum_{n}:\left|\arg \left\{-\frac{z F^{\prime}(\mathrm{z})}{F(\mathrm{z})}\right\}\right|<\frac{\alpha \pi}{2}, z \in \dot{U}\right\}
$$

the class of meromorphic-strongly starlike functions of order.

Let $\mathrm{p}$ be positive integer and let $\mathrm{A}(\mathrm{p})$ be the class of functions

$$
f(\mathrm{z})=\mathrm{z}^{\mathrm{p}}+\sum_{n=p+1}^{\infty} c_{n} z^{n},
$$

which are analytic in the unit disk $D=\{z \in \mathbb{C}:|z|<1\}$ Furthermore, denote by $A$ the class of analytic functions in $\mathrm{D}$ and usually normalized, i.e. $A=\left\{f \in H: f(0), f^{\prime}(0)\right\}$ We say that the $f € H$ is subordinate to $g € H$ in the unit disc $\mathrm{D}$, written $f \prec g$ if and only if there exists an analytic function $\mathrm{w} € \mathrm{H}$ such that and $\mathrm{f}(\mathrm{z})=\mathrm{g}[\mathrm{w}(\mathrm{z})]$ for $\mathrm{z} \in \mathrm{D}$. Therefore $f \prec g$ in $D$ implies $\mathrm{f}(\mathrm{D}) \subset \mathrm{g}(\mathrm{D})$. In particular if $\mathrm{g}$ is univalent in $\mathrm{D}$ then the Subordination Principle says that $f \prec g$ if and only if $\mathrm{f}(0)=\mathrm{g}(0)$ and $\mathrm{f}(|\mathrm{z}|<\mathrm{r}) \mathrm{g}(|\mathrm{z}|<\mathrm{r})$, for all $\mathrm{r} €(0,1]$.

The subclass of $\mathrm{A}(\mathrm{p})$ consisting of $\mathrm{p}$-valently starlike functions is denoted by $S^{\star}(p)$. An analytic description of $S^{\star}(p)$ is given by

$$
\mathrm{S}^{*}(\mathrm{p})=\left\{f \in A(\mathrm{p}):\left|\arg \frac{z f^{\prime}(\mathrm{z})}{f(\mathrm{z})}\right|<\frac{\pi}{2}, z \in D\right\}
$$

The subclass of $\mathrm{A}(\mathrm{p})$ consisting of $\mathrm{p}$-valently and strongly starlike functions of order $\alpha, 0<\alpha \leq 1$ is denoted by $\mathrm{S}^{\star} \alpha(\mathrm{p})$. An analytic description of $S^{*}(\mathrm{p})$ is given by

$$
\mathrm{S}_{\alpha}^{*}(\mathrm{p})=\left\{f \in A(\mathrm{p}):\left|\arg \frac{z f^{\prime}(\mathrm{z})}{f(\mathrm{z})}\right|<\frac{\alpha \pi}{2}, z \in D\right\}
$$

The subclass of $\mathrm{A}(\mathrm{p})$ consisting of $\mathrm{p}$-valently convex functions and $p$-valently strongly convex functions of order $\alpha, 0<\alpha \leq 1$ are denoted by $\mathrm{C}^{\star}(\mathrm{p})$ and $\mathrm{C}^{\star}(\mathrm{p})$ respectively. The analytic descriptions of $\mathrm{C}^{\star}(\mathrm{p})$ and $\mathrm{C}^{*}(\mathrm{p})$ are given by

$$
\mathrm{C}^{*}(\mathrm{p})=\left\{f \in A(\mathrm{p}):\left|\arg \left\{1+\frac{z f^{\prime \prime}(\mathrm{z})}{f^{\prime}(\mathrm{z})}\right\}\right|<\frac{\pi}{2}, z \in D\right\}
$$

and

$$
\mathrm{C}_{\alpha}^{*}(\mathrm{p})=\left\{f \in A(\mathrm{p}):\left|\arg \left\{1+\frac{z f^{\prime \prime}(\mathrm{z})}{f^{\prime}(\mathrm{z})}\right\}\right|<\frac{\alpha \pi}{2}, z \in D\right\}
$$

\section{Main Result}

To prove the main results, we also need the following generalization of Nunokawa's lemma [1-12]. Lemma 2.1: [5] Let $\mathrm{p}(\mathrm{z})$ be of the form

$$
p(\mathrm{z})=1+\sum_{n=m \geq 1}^{\infty} a_{n} z^{n}, a_{m} \neq 0,(|z|<1),
$$

with $\mathrm{p}(\mathrm{z}) 6 \neq 0$ in $|\mathrm{z}|<1$. If there exists a point $\mathrm{z}_{0},\left|\mathrm{z}_{0}\right|<1$, such that

$|\arg \{\mathrm{p}(\mathrm{z})\}|<\pi \alpha / 2$ in $|\mathrm{z}|<\left|\mathrm{z}_{0}\right|$

And

$\arg \left\{\mathrm{p}\left(\mathrm{z}_{0}\right)\right\} \mid=\pi \alpha / 2$

for some $\alpha>0$, then we have

$\frac{\mathrm{z}_{0} \mathrm{p}^{\prime}\left(\mathrm{z}_{0}\right)}{p\left(\mathrm{z}_{0}\right)}=i k \alpha$,

Where

*Corresponding author: Janusz Sokol, Department of Mathematics, Rzesz'ow University of Technology, Al. Powsta'nc'ow Warszawy 12, 35-959 Rzesz'ow Poland, Tel: 81-27-220-7111; E-mail: jsokol@prz.edu.pl

Received January 06, 2014; Accepted March 28, 2014; Published April 07, 2014

Citation: Sokol J, Nunokawa M (2014) On Certain Analytic Functions. J Appl Computat Math 3: 159 doi:10.4172/2168-9679.1000159

Copyright: (c) 2014 Sokol J, et al. This is an open-access article distributed under the terms of the Creative Commons Attribution License, which permits unrestricted use, distribution, and reproduction in any medium, provided the original author and source are credited. 
$\mathrm{k} \geq \mathrm{m}\left(\alpha^{2}+1\right) /(2 \mathrm{a}) \quad$ when $\arg \left\{\mathrm{p}\left(\mathrm{z}_{0}\right)\right\}=\pi \alpha / 2$ and

$\mathrm{k} \geq-\mathrm{m}\left(\alpha^{2}+1\right) /(2 \mathrm{a}) \quad$ when $\arg \left\{\mathrm{p}\left(\mathrm{z}_{0}\right)\right\}=-\pi \alpha / 2$

Where

$\left\{\mathrm{p}\left(\mathrm{z}_{0}\right)\right\}^{1 / \alpha}= \pm \mathrm{ia}, \mathrm{a}>0$

Theorem 3.1: Let $\mathrm{p}(\mathrm{z})$ be analytic in $\mathrm{D}$ with $\mathrm{p}(0)-1=\mathrm{p}^{\prime}(0)=0$. Assume that

$\alpha €[0,1 / 2]$. If for $z € D$

$\left|\arg \left\{\mathrm{p}(\mathrm{z})-\mathrm{zp}^{\prime}(\mathrm{z})\right\}\right|<\arctan (2 \alpha)-\frac{\alpha \pi}{2}$,

then

$|\arg \{\mathrm{p}(\mathrm{z})\}|<\frac{\alpha \pi}{2} \quad(\mathrm{z} \in \mathrm{D})$

Proof: If there exists a point $z_{0},\left|z_{0}\right|<1$, such that

$|\arg \{\mathrm{p}(\mathrm{z})\}|<\pi \alpha / 2\left(|z| \leq\left|z_{0}\right|\right)$

and

$|\arg \{\mathrm{p}(\mathrm{z})\}|=<\pi \alpha / 2$,

then from Nunokawa's lemma 2.1, with $\mathrm{m}=2$, we have

$$
\frac{\mathrm{z}_{0} \mathrm{p}^{\prime}\left(\mathrm{z}_{0}\right)}{p\left(\mathrm{z}_{0}\right)}=i k \alpha,
$$

where $\mathrm{k}$ is a real number

$\mathrm{k} \geq\left(\mathrm{a}^{2}+1\right) / \mathrm{a}$, when $\arg \left\{\mathrm{p}\left(\mathrm{z}_{0}\right)\right\}=\pi \alpha / 2$

And

$\mathrm{k} \leq-\left(\mathrm{a}^{2}+1\right) / \mathrm{a}$, when $\arg \left\{\mathrm{p}\left(\mathrm{z}_{0}\right)\right\}=-\pi \alpha / 2$

And where $p\left(z_{0}\right)^{1 / \alpha}= \pm i a, a>0$. For the case $\arg \left\{\mathrm{p}\left(\mathrm{z}_{0}\right)\right\}=$ $\pi \alpha / 2$, we have

$$
p\left(z_{0}\right)-z_{0} p^{\prime}\left(z_{0}\right)=p\left(z_{0}\right)\left(1-\frac{z_{0} p^{\prime}\left(z_{0}\right)}{p\left(z_{0}\right)}\right)=(i a)^{\alpha}(\mathrm{i}-\alpha k)
$$

where $\mathrm{k} \geq\left(\mathrm{a}^{2}+1\right) / \mathrm{a}$ and $0<\mathrm{a}$. Because, $\mathrm{k} \geq\left(\mathrm{a}^{2}+1\right) / \mathrm{a} \geq 2$, we have

$$
-\frac{\pi}{2}<\arg (1-i \alpha k) \leq-\arctan (2 \alpha)
$$

It is easy to see that for $\alpha €[0,1 / 2]$ we have

$$
\arctan (2 \alpha)-\frac{\alpha \pi}{2} \geq 0
$$

Therefore, using (3.3) and (3.4), we obtain

$$
\begin{array}{r}
\arg \left\{p\left(\mathrm{z}_{0}\right)-\mathrm{z}_{0} p^{\prime}\left(\mathrm{z}_{0}\right)\right\}=\arg \left\{p\left(\mathrm{z}_{0}\right)\right\}+\arg \left\{1-\frac{\mathrm{z}_{0} \mathrm{p}^{\prime}\left(\mathrm{z}_{0}\right)}{\mathrm{p}\left(\mathrm{z}_{0}\right)}\right\} \\
=\arg \left\{(\mathrm{ia})^{\alpha}\right\}+\arg \{1-i \alpha k\} \\
\leq-\left\{\arctan (2 \alpha)-\frac{\alpha \pi}{2}\right\}
\end{array}
$$

This is a contradiction with (3.1). For the case $\arg \left\{p\left(z_{0}\right)\right\}=-\pi \alpha / 2$, we have

$$
p\left(\mathrm{z}_{0}\right)-\mathrm{z}_{0} p^{\prime}\left(\mathrm{z}_{0}\right)=p\left(z_{0}\right)\left(1-\frac{\mathrm{z}_{0} \mathrm{p}^{\prime}\left(\mathrm{z}_{0}\right)}{\mathrm{p}\left(\mathrm{z}_{0}\right)}\right)
$$

$$
=(-i a)^{\alpha}(1-\mathrm{i} \alpha \mathrm{k}) \text {, }
$$

where $k \leq-\left(a^{2}+1\right) / a \leq-2$. We also have

$$
\arctan (2 \alpha) \leq \arg (1-\mathrm{i} \alpha \mathrm{k})<\frac{\pi}{2}
$$

Therefore, using (3.6) and (3.7) and applying the same method as above, we obtain

$$
\begin{aligned}
& \arg \left\{p\left(\mathrm{z}_{0}\right)-\mathrm{z}_{0} p^{\prime}\left(\mathrm{z}_{0}\right)\right\}=\arg \left\{p\left(z_{0}\right)\right\}+\arg \left\{1-\frac{\mathrm{z}_{0} \mathrm{p}^{\prime}\left(\mathrm{z}_{0}\right)}{\mathrm{p}\left(\mathrm{z}_{0}\right)}\right\} \\
& =\arg \left\{(-\mathrm{ia})^{\alpha}\right\}+\arg (1-\mathrm{i} \alpha \mathrm{k}) \geq \arctan (2 \alpha)-\frac{\alpha \pi}{2}
\end{aligned}
$$

This is also a contradiction with (3.1), and it completes the proof.

Let us put $p(\mathrm{z})=\mathrm{e}^{-\mathrm{i} \beta} q\left(\mathrm{e}^{\mathrm{i} \beta} \mathrm{z}\right)$ in Theorem 3.1.

Corollary 3.2: Let $p(\mathrm{z})=\mathrm{e}^{-\mathrm{i} \beta} q\left(\mathrm{e}^{\mathrm{i} \beta} \mathrm{z}\right), \beta \in \mathbb{R}$, be analytic in $\mathrm{D}$ with $\mathrm{p}(0)-1=\mathrm{p}^{\prime}(0)=0$. Assume

that $\alpha €[0,1 / 2]$. If for $z € D$

$$
\left|\arg \left\{\mathrm{e}^{-\mathrm{i} \beta} q\left(\mathrm{e}^{\mathrm{i} \beta} \mathrm{z}\right)-z q^{\prime}\left(\mathrm{e}^{\mathrm{i} \beta} \mathrm{z}\right)\right\}\right|<\arctan (2 \alpha)-\frac{\alpha \pi}{2},
$$
then

$$
\left|\arg \left\{\mathrm{e}^{-\mathrm{i} \beta} q\left(\mathrm{e}^{\mathrm{i} \beta} \mathrm{z}\right)\right\}\right|<\frac{\alpha \pi}{2} \quad(\mathrm{z} \in D)
$$

Corollary 3.3: Let $p(\mathrm{z})=\mathrm{e}^{-\mathrm{i} \beta} q\left(\mathrm{e}^{\mathrm{i} \beta} \mathrm{z}\right), \beta \in \mathbb{R}$, be analytic in $\mathrm{D}$ with $\mathrm{p}(0)-1=\mathrm{p}^{\prime}(0)=0$. Assume

that $\alpha €[0,1 / 2]$. If for $z € D$

$$
\begin{aligned}
& \left|\arg \left\{q\left(\mathrm{e}^{\mathrm{i} \beta} \mathrm{z}\right)-\mathrm{e}^{\mathrm{i} \beta} z q^{\prime}\left(\mathrm{e}^{\mathrm{i} \beta} z\right)\right\}-\beta\right|<\arctan (2 \alpha)-\frac{\alpha \pi}{2} \\
& \left|\arg \left\{q\left(\mathrm{e}^{\mathrm{i} \beta} \mathrm{z}\right)\right\}-\beta\right|<\frac{\alpha \pi}{2} \quad(\mathrm{z} \in \mathrm{D})
\end{aligned}
$$

Corollary 3.4: Let $\mathrm{q}(\mathrm{z})$ be analytic in $D$ with $\mathrm{q}(0)=\mathrm{e}^{\mathrm{i} \beta}, \mathrm{q}^{\prime}(0)=0, \beta €$ $\mathrm{R}$. Assume that $\alpha €[0,1 / 2]$. If for $\mathrm{z} € \mathrm{D}$

$$
\begin{aligned}
& \left|\arg \left\{q(\mathrm{z})-z q^{\prime}(z)\right\}-\beta\right|<\arctan (2 \alpha)-\frac{\alpha \pi}{2} \\
& \mid \arg \left\{q(\mathrm{z})-\beta \mid<\frac{\alpha \pi}{2} \quad(\mathrm{z} \in \mathrm{D})\right.
\end{aligned}
$$

Theorem 3.5: Let

$$
F(\mathrm{z})=\frac{1}{z}+a_{1} z+a_{2} z^{2}+\ldots, \quad z \in U
$$

Assume that $\alpha €[0,1 / 2]$. If for $\mathrm{z} € D$

$$
\left|\arg \left\{-z^{2} F^{\prime}(\mathrm{z})\right\}\right|<\arctan (2 \alpha)-\frac{\alpha \pi}{2}
$$

then

$$
|\arg \{z F(\mathrm{z})\}|<\frac{\alpha \pi}{2} \quad(\mathrm{z} \in \mathrm{D})(3.16)
$$

Proof: Let

$\mathrm{p}(\mathrm{z})=\mathrm{zF}(\mathrm{z})=1+\mathrm{a}_{1} \mathrm{z}^{2}+\mathrm{a}_{2} \mathrm{z}^{3}+\ldots, \quad \mathrm{p}(0)=1$ 
Then

$$
p(z)-z p^{\prime}(z)=-z^{2} F^{\prime}(z) \text {. }
$$

Applying Theorem 3.1 we obtain the result.

\section{Corollary 3.6: Let}

$$
\mathrm{F}(\mathrm{z})=\frac{1}{z}+\mathrm{a}_{1} \mathrm{z}+\mathrm{a}_{2} \mathrm{z}^{2}+\ldots, \quad \mathrm{z} \in \mathrm{U}
$$

Assume that $\alpha €[0,1 / 2]$. If for $z € D$

$$
\left|\arg \left\{-z^{2} F^{\prime}(\mathrm{z})\right\}\right|<\arctan (2 \alpha)-\frac{\alpha \pi}{2}
$$

then $\mathrm{F}(\mathrm{z})$ is meromorphic-strongly starlike function of order arctan $(2 \alpha)$.

Proof: For showing that $\mathrm{F}(\mathrm{z})$ is meromorphic-strongly starlike function we need to show (1.1). Applying theorem 3.5 and 3.17 we obtain $|\arg \{z F(z)\}|<\alpha \pi / 2$, since this and since (3.17), we obtain

$$
\begin{aligned}
& \left|\arg \frac{\left\{-z F^{\prime}(z)\right\}}{F(\mathrm{z})}\right| \\
& =\left|\arg \frac{\left\{-z^{2} F^{\prime}(z)\right\}}{z F(\mathrm{z})}\right| \\
& \leq\left|\arg \left\{-z^{2} F^{\prime}(z)\right\}\right|+|\arg \{\mathrm{zF}(\mathrm{z})\}| \\
& <\arctan (2 \alpha)-\frac{\alpha \pi}{2}+\frac{\alpha \pi}{2} \\
& =\arctan (2 \alpha)
\end{aligned}
$$

Therefore, $\mathrm{F}(\mathrm{z})$ is meromorphic-strongly starlike function of order $\arctan (2 \alpha)$.

For $\alpha[0,1 / 2]$ we have

$$
0 \leq \arctan (2 \alpha)-\frac{\alpha \pi}{2} \leq \arctan \left(2 \alpha_{0}\right)-\frac{\alpha_{0} \pi}{2}
$$

Where

$$
\alpha_{0}=\sqrt{\frac{4-\pi}{4 \pi}}=0.26 \ldots
$$

\section{Theorem 3.7: Let}

$$
\mathrm{F}(\mathrm{z})=\frac{1}{z}+\mathrm{a}_{1} \mathrm{z}+\mathrm{a}_{2} \mathrm{z}^{2}+\ldots, \quad \mathrm{z} \in \mathrm{U}
$$

Assume that $\alpha €[0,1 / 2]$. If for $z € D$

$$
\left|\arg \left\{\frac{z^{2}\left(\mathrm{~F}^{\prime \prime}(\mathrm{z})-\left(\mathrm{F}^{\prime}(\mathrm{z})\right)^{2}\right)}{F^{2}(\mathrm{z})}\right\}\right|<\arctan (2 \alpha)-\frac{\alpha \pi}{2}
$$

then

$\left|\arg \left\{\frac{z \mathrm{~F}^{\prime}(\mathrm{z})}{F(\mathrm{z})}\right\}\right|<\frac{\alpha \pi}{2} \quad(\mathrm{z} \in \mathrm{D})$

Proof: Let $\mathrm{p}(\mathrm{z})=\mathrm{zF}(\mathrm{z}) / \mathrm{F}(\mathrm{z})$. By (3.18) we have that

$\mathrm{p}(\mathrm{z})=1+\mathrm{p}_{2} \mathrm{z}^{2}+\mathrm{p}_{3} \mathrm{z}^{3}+\ldots$

Moreover

$\mathrm{p}(\mathrm{z})-z p^{\prime}(\mathrm{z})=\frac{z^{2}\left(\mathrm{~F}^{\prime \prime}(\mathrm{z})-\left(F^{\prime}(\mathrm{z})\right)^{2}\right)}{F^{2}(\mathrm{z})}$

Applying Theorem 3.1 we obtain the result.

\section{References}

1. Brannan DA, Kirwan WE (1969) On some classes of bounded univalent functions. J London Math Soc 1: 431-443.

2. Fukui S, Sakaguchi K (1980) An extension of a theorem of S Ruscheveyh. Bill Fac Edu Wakayama Univ Nat Sci 29: 1-3.

3. Nunokawa M (1992) On Properties of Non-Carath'eodory Functions. Proc Japan Acad 68 Ser A 152-153.

4. Nunokawa M (1993) On the Order of Strongly Starlikeness of Strongly Convex Functions. Proc Japan Acad 69 Ser: 234-237.

5. Nunokawa M, Sokol J New conditions for starlikeness and strongly starlikeness of order alpha.

6. Nunokawa M, Sokol J (2012) On some sufficient conditions for univalence and starlikeness. J Ineq Appl 282.

7. Nunokawa M, Sokol J (2013) On the order of strongly starlikeness of convex functions of order alpha. Mediterranean J Math.

8. Robertson MS (1936) On the theory of univalent functions. Ann Math 37: 374 408.

9. Sokol J, Trojnar-Spelina L (2013) On a sufficient condition for strongly starlikeness. J Ineq Appl 2013: 383

10. Stankiewicz J (1966) Quelques probl'emes extr'emaux dans les classes des functions -angulairement 'etoil'ees, Ann Univ Mariae Curie-Sk lodowska, Sect A 20: $59-75$.

11. Strrohh"acker E (1933) Beitrage z"ur Theorie der schlichter Functinen. Math Z 37: 356-380.

12. Wilken DR, Feng J (1980) A remark on convex and starlike functions. J London Math Soc 21: 287-290. 